\title{
Anxiety sensitivity as a predictor of outcome in the treatment of obsessive-compulsive disorder
}

\author{
Shannon M. Blakey ${ }^{a,}{ }^{*}$, Jonathan S. Abramowitz ${ }^{a}$, Lillian Reuman ${ }^{\mathrm{a}}$, Rachel C. Leonard ${ }^{\mathrm{b}}$, \\ Bradley C. Riemann ${ }^{\mathrm{b}}$ \\ ${ }^{a}$ University of North Carolina at Chapel Hill, Chapel Hill, NC, United States \\ ${ }^{\mathrm{b}}$ Rogers Memorial Hospital, Oconomowoc, WI, United States
}

\section{A R T I C L E I N F O}

\section{Article history:}

Received 8 April 2016

Received in revised form

20 February 2017

Accepted 2 May 2017

Available online 6 May 2017

\begin{abstract}
A B S T R A C T
Background and objectives: To address the fact that not all individuals who receive cognitive-behavioral therapy (CBT) for obsessive-compulsive disorder (OCD) exhibit complete symptom reduction, research has examined factors that predict outcome; however, no studies have examined anxiety sensitivity (AS) as a predictor of outcome of CBT for OCD. AS refers to the fear of anxious arousal that results from mistaken beliefs about the dangerousness of anxiety-related body sensations. It is important to understand whether AS influences OCD treatment outcome, considering that (a) some obsessions directly relate to AS, and (b) OCD patients with high AS may be reluctant to engage in anxiety-provoking components of CBT for OCD.

Methods: Patients $(N=187)$ with a primary diagnosis of OCD who received residential CBT for OCD participated in this study, which involved completing a self-report battery at pre- and post-treatment. Results: Results supported study hypotheses, in that (a) baseline AS positively correlated with baseline OCD severity, and (b) greater baseline AS prospectively predicted higher posttreatment OCD symptom severity even after controlling for pretreatment OCD and depression severity.

Limitations: The study was limited by its use of an older measure of AS, reliance on self-report measures, and nonstandardized treatment across participants.

Conclusions: Findings highlight the importance of AS in the nature and treatment of OCD. Clinical implications and future directions are discussed.
\end{abstract}

() 2017 Elsevier Ltd. All rights reserved.
Obsessive-compulsive disorder (OCD) is characterized by distressing, unwanted intrusive thoughts, images, and doubts (i.e., obsessions) and/or urges to perform repetitive, deliberate rituals and other anxiety-reduction strategies to neutralize this distress (i.e., compulsions) (American Psychiatric Association [APA], 2013). Compulsive rituals are reinforced by the immediate decrease in anxiety they engender, yet the anxiety reduction is temporary and compulsions prevent the natural extinction of obsessional fear in the long term. Obsessive-compulsive symptoms cause considerable distress and functional impairment among the $2-3 \%$ of the population that experiences OCD at some point in their lifetime (Kessler et al., 2005).

Cognitive-behavioral therapy (CBT) using exposure and

\footnotetext{
* Corresponding author. University of North Carolina at Chapel Hill, C.B. \# 3270 (Davie Hall), Chapel Hill, NC 27599, United States.

E-mail address: sblakey@unc.edu (S.M. Blakey).
}

response prevention (ERP) procedures can be an effective treatment for OCD (e.g., Olatunji, Cisler, \& Deacon, 2010). Exposure entails repeated systematic confrontation with situations and stimuli that provoke obsessional anxiety; response prevention involves resisting urges to perform escape and avoidance behaviors (e.g., compulsive rituals) during and after exposure trials. Yet despite its established efficacy, response to this intervention varies widely and some individuals are not able to adhere or respond. Accordingly, researchers have sought to identify factors that predict outcome. Severe depression and baseline OCD symptoms, for example, have been associated with attenuated response to $\mathrm{CBT}$ for OCD across a large body of research (Abramowitz \& Foa, 2000; Abramowitz, Franklin, Kozak, Street, \& Foa, 2000; Farrell et al., 2016; Foa et al., 1983; Knopp, Knowles, Bee, Lovell, \& Bower, 2013; Steketee, Chambless, \& Tran, 2001), although the relationship between baseline depression and OCD treatment outcome is not consistent and does not appear to influence outcomes in the 
long-term (e.g., Anholt et al., 2011).

To date, no studies have examined anxiety sensitivity (AS) as a predictor of CBT outcome in OCD. AS refers to mistaken beliefs about the dangerousness of anxiety-related body sensations that generate the fear of anxious arousal (e.g., Reiss \& McNally, 1985). Specifically, individuals with elevated AS are hypervigilant to and (mis)appraise ambiguous body sensations as particularly dangerous. For example, someone with high anxiety sensitivity might misinterpret chest tightness as a sign of a heart attack, dizziness as a sign of "losing control," or racing thoughts as an indicator that one is "going crazy" and about do something embarrassing or harmful. Although AS is often associated with panic disorder, it is considered a transdiagnostic process (Taylor, 1999); moreover, AS has demonstrated moderate to strong associations with OCD symptom severity in individuals diagnosed with OCD (Pearson $r$ s range 0.28-0.30; partial $r$ s range 0.28-0.64; Calamari, Rector, Woodard, Cohen, \& Chik, 2008; Deacon \& Abramowitz, 2006; Laposa, Collimore, Hawley, \& Rector, 2015; Norton, Sexton, Walker, \& Norton, 2005; Zinbarg, Barlow, \& Brown, 1997; for a review see Robinson \& Freeston, 2014) as well among nonclinical individuals (Pearson rs range 0.30-0.56; David et al., 2009; Keough, Riccardi, Timpano, Mitchell, \& Schmidt, 2010; Sexton, Norton, Walker, \& Norton, 2003; Wheaton, Deacon, McGrath, Berman, \& Abramowitz, 2012).

Although research in this area is limited, a few studies have highlighted relationships between specific dimensions of AS (i.e., the fear of anxiety and/or anxious arousal because of feared physical, mental, or social consequences) and OCD symptoms (e.g., Wheaton, Mahaffey, Timpano, Berman, \& Abramowitz, 2012). Previous investigators have posited that the cognitive dimension of AS in particular is strongly related to OCD given that misappraisals of one's own thinking (e.g., overestimating the importance of thoughts) are a cardinal feature of OCD (Rachman, 1997, 1998). Although some research supports this hypothesis (e.g., Cox, Borger, \& Enns, 1999; Sexton et al., 2003; Wheaton et al., 2012), aggregate findings are inconsistent (Deacon \& Abramowitz, 2006). Moreover, the cross-sectional nature of previous studies precludes investigators from determining whether AS predicts changes in OCD symptom severity over time (e.g., following treatment).

There are a number of reasons to examine AS as a predictor of poorer outcome with CBT for OCD. First, anxious arousal is often provoked during exposure therapy. Thus, individuals with high AS are apt to become afraid not only of exposure stimuli per se, but also the arousal sensations induced when conducting exposures. Given that AS is a strong predictor of panic attacks (e.g., Schmidt, Zvolensky, \& Maner, 2006), OCD patients with high AS might be prone to experiencing panic episodes during exposure, which could lead to avoidance behavior and hamper both adherence and confidence in the treatment techniques, thereby leading to suboptimal outcome. Furthermore, some patients' obsessions might directly relate to ambiguous somatic sensations; for example, a patient who presents with contamination-related OCD may be especially hypervigilant for (and anxious in response to) feelings of nausea, which often accompany anxious arousal.

One methodological obstacle to examining predictors of outcome in OCD is that patients with high levels of concurrent anxiety and depression (i.e., comorbidity) are often excluded from randomized controlled trials (RCTs) in efforts to maximize the internal validity of such studies. Yet this restricts the range of comorbid psychopathology variables (e.g., depression, AS), potentially obscuring relationships in secondary analyses of outcome predictors. We addressed this issue in the present investigation by using a large sample of individuals with OCD seeking treatment outside the context of an RCT. Our data were collected within a residential program specializing in CBT for OCD in which individuals often met criteria for comorbid conditions (e.g., major depression) and were concurrently using psychotropic medication. Although these sample characteristics might attenuate internal validity (e.g., treatment was less standardized than in controlled studies), it afforded the best opportunity to observe the effects of AS on CBT response in a diverse OCD clinical sample receiving CBT in a service setting.

The present study was designed to examine the extent to which baseline levels of AS predict treatment outcome in a sample of individuals with a clinical diagnosis of OCD undergoing CBT above and beyond established predictors of attenuated response (i.e., baseline OCD and depressive symptom severity). On the basis of previous research and the conceptual considerations noted above, we expected that baseline AS would be positively correlated with baseline OCD severity. We also hypothesized that greater baseline levels of AS would prospectively predict higher posttreatment OCD symptom severity above and beyond pretreatment OCD and depression severity.

\section{Method}

\subsection{Participants}

Participants were considered eligible to participate in the current study if they (a) had a primary diagnosis of $O C D$, (b) were admitted for residential treatment at the Obsessive-Compulsive Disorders Center at Rogers Memorial Hospital (RMH) in Oconomowoc, Wisconsin, (c) provided informed consent to participate, and $(\mathrm{d})$ had completed pre- and post-treatment assessments. Patients with comorbid psychotic symptoms or current substance abuse were considered ineligible. On average, approximately $28.5 \mathrm{~h}$ of ERP were completed per week, including both staff-assisted and self-directed exposure practice. Most participants $(n=163 ; 87.2 \%)$ were also taking psychiatric medications; primarily, selective serotonin reuptake inhibitors (SSRIs; $n=125$; 66.8\%). Psychiatric medications were adjusted upon the on-site psychiatrist's assessments of the patient's needs (information on the precise number of participants whose medication changed during the course of CBT was unfortunately not available). The majority $(n=153 ; 81.8 \%)$ had secondary diagnoses, which are shown in Table 1.

The final sample included 187 adults (51.9\% women; $n=97$ ) who had a mean age of 30.49 years $(S D=12.24)$ and had received

Table 1

Secondary diagnosis of study participants.

\begin{tabular}{lll}
\hline Secondary diagnosis & $n$ & $(\%)$ \\
\hline Mood disorders & 62 & $(33.2)$ \\
Major depressive disorder & 10 & $(5.3)$ \\
Bipolar I disorder & 1 & $(0.5)$ \\
Other mood disorder & 51 & $(27.3)$ \\
Anxiety-related disorders & 36 & $(19.3)$ \\
Panic disorder & 4 & $(2.1)$ \\
Posttraumatic stress disorder & 1 & $(0.5)$ \\
Social anxiety disorder & 10 & $(5.3)$ \\
General anxiety disorder & 21 & $(11.2)$ \\
Obsessive-compulsive and related disorders & 5 & $(2.7)$ \\
Trichotillomania & 1 & $(0.5)$ \\
Tic disorder/Tourette's syndrome & 1 & $(0.5)$ \\
Body dysmorphic disorder & 3 & $(1.6)$ \\
Developmental disorders & 2 & $(1.1)$ \\
Attention deficit/hyperactivity disorder & 1 & $(0.5)$ \\
Learning disability & 1 & $(0.5)$ \\
Eating disorders & 3 & $(1.6)$ \\
Anorexia nervosa & 1 & $(0.5)$ \\
Bulimia nervosa & 1 & $(0.5)$ \\
Other eating disorder & 1 & $(0.5)$ \\
Other psychological disorder & 45 & $(24.1)$ \\
\hline
\end{tabular}


an average of 14.93 years $(S D=2.29)$ of education. The majority of participants $(n=167 ; 89.3 \%)$ identified as White/European American, with $3.7 \%(n=7)$ identifying as Asian and $1.1 \%(n=2)$ identifying as African American. Eight (4.3\%) participants self-identified as being of Hispanic origin. Three participants (1.6\%) did not selfdisclose their race/ethnicity. Most participants ( $n=128 ; 68.4 \%)$ were single; $24.6 \%(n=46)$ were married and $2.1 \%(n=4)$ were divorced (marital status was undocumented for two participants).

\subsection{Procedure}

\subsubsection{Assessment}

Prior to admission to the residential treatment program, all prospective patients completed an initial assessment with a trained intake staff member. This included the clinician-rated version of the semi-structured Yale-Brown Obsessive-Compulsive Scale (Y-BOCS; Goodman, Price, Rasmussen, \& Mazure, 1989a, 1989b) symptom checklist and severity rating to determine the presence of DSM-IV OCD (APA, 2000). The clinical director of the ObsessiveCompulsive Disorder Center ([AUTHOR INITIALS]) reviewed the results of this assessment with the intake interviewer and determined if the patient was appropriate for admission. Individuals were only admitted to the clinic for treatment, and thus included in the current study, if there was $100 \%$ diagnostic agreement between the intake interviewer and clinical director. Each participant also completed a self-report assessment packet that included the study measures described further below, which was re-administered at posttreatment. As part of the admissions process, patients provided consent to allow their responses to the study measures to be used for both clinical and research purposes. The consent procedures and study measures were approved by both the RMH Human Subjects Committee and the Rogers Center for Research and Training.

\subsubsection{Treatment}

Treatment consisted of ERP, which followed the procedures described by Kozak and Foa (1997). Therapists and participants developed a list of situations for exposure that triggered their anxiety from least to most feared and then assisted participants in facing these feared situations in a prolonged, repetitive, and graduated manner while at the same time helping them to resist engaging in avoidance behaviors and/or rituals. Participants also engaged in cognitive restructuring to help them examine and disconfirm their irrational beliefs. Additional interventions (e.g., behavioral activation for symptoms of depression) were often used to address comorbid symptoms. Further, participants met regularly with their therapist for non-CBT work (e.g., psychoeducation with family members), participated in a process group once per week, and participated in experiential therapy groups several times per week.

\subsection{Measures}

\subsubsection{Dimensional Obsessive-Compulsive Scale (DOCS; Abramowitz et al., 2010)}

The DOCS is a 20-item self-report measure with four empirically derived subscales that assesses the severity of the four most consistently replicated OCD symptom dimensions: (a) contamination (DOCS-C), (b) responsibility for harm and mistakes (DOCS-H), (c) unacceptable thoughts (DOCS-UT), and (d) symmetry/ordering (DOCS-S). Each subscale begins with a description of the symptom dimension as well as prototypical examples of fears, rituals, and avoidance behaviors. Next, within each symptom dimension, five items (rated 0 to 4 ) assess the following parameters of severity: (a) time occupied by obsessions and rituals, (b) avoidance behavior, (c) associated distress, (d) functional interference, and (e) difficulty disregarding the obsessions and refraining from the compulsions. The DOCS has demonstrated excellent reliability in clinical samples $(\alpha=0.94-0.96)$, and the measure converges well with other measures of OCD symptoms (Abramowitz et al., 2010).

\subsubsection{Beck Depression Inventory-II (BDI-II; Beck, Steer, \& Brown, 1996)}

The BDI-II is a 21-item self-report scale that assesses the severity of affective, cognitive, motivational, vegetative, and psychomotor components of depression. Scores of 10 or less are considered normal; scores of 20 or greater suggest the presence of clinical depression. The BDI-II has excellent reliability and validity and is widely used in clinical research (Beck et al., 1996).

\subsubsection{Anxiety Sensitivity Index (ASI; Reiss, Epstein, \& Gursky, 1986)}

The ASI is a unidimensional 16-item self-report measure of beliefs regarding the dangerousness of anxious arousal (e.g., "It scares me when my heart beats rapidly"). Participants rate their agreement with each statement on a 0 (very little) to 4 (very much) scale; higher scores indicate greater AS. The ASI has demonstrated adequate reliability and validity in previous research (Peterson \& Reiss, 1992; Reiss, Peterson, Gursky, \& McNally, 1986). Three ASI subscales were computed according to the principal components solution recommended by Taylor, Koch, Woody, and McLean (1996): ASI-Physical Concerns (items 6, 7, 8, 9, 10, and 11; Cronbach $\alpha=0.81$ ), ASI-Mental Concerns (items 2, 12, and 15; Cronbach $\alpha=0.80$ ), and ASI-Social Concerns (items, 1, 3, 4, 5, 13, 14, and 16; Cronbach $\alpha=0.75$ ).

\section{Results}

Data were first screened to assess concordance with statistical assumptions. Distributions of scores on study measures were free of significant skew and kurtosis (all values $<2$ ). DOCS, ASI, and BDIII scores fell within the range of expected values. The sample exhibited clinically significant baseline AS $(M=23.94 ; S D=12.28)$ and depressive symptoms $(M=26.28 ; S D=13.47)$ prior to treatment. The sample's mean DOCS total score indicated clinically significant OCD symptom severity prior to treatment $(M=32.73$; $S D=15.07)$. At posttreatment, the mean DOCS total score was 16.59 $(S D=11.74)$. A paired-samples $t$-test revealed that the change in DOCS total scores from pre-to posttreatment was significant, $t(123)=13.22, p<0.001, d=1.18$.

To test our first hypothesis that AS would be associated with baseline OCD symptoms, we computed zero-order correlations between ASI total and subscale scores and pretreatment DOCS total scores. Correlations among study variables are presented in Table 2. These analyses revealed significant positive correlations among pretreatment DOCS and ASI total and subscale scores (all ps $<0.001)$.

\section{Table 2}

Zero-Order bivariate correlations among study variables.

\begin{tabular}{lllllll}
\hline Variable & 1 & 2 & 3 & 4 & 5 & 6 \\
\hline 1. Posttreatment DOCS & - & & & & & \\
2. Baseline DOCS & 0.52 & - & & & & \\
3. Baseline BDI-II & 0.26 & 0.36 & - & & & \\
4. Baseline ASI-Total & 0.45 & 0.39 & 0.42 & - & & \\
5. Baseline ASI-Physical & 0.41 & 0.31 & 0.26 & 0.86 & - & \\
6. Baseline ASI-Mental & 0.35 & 0.27 & 0.48 & 0.76 & 0.45 & - \\
7. Baseline ASI-Social & 0.35 & 0.40 & 0.41 & 0.92 & 0.67 & 0.66
\end{tabular}

DOCS $=$ Dimensional Obsessive-Compulsive Scale; BDI-II = Beck Depression Inventory-II; ASI = Anxiety Sensitivity Index; all $p s<0.01$. 
To test our second hypothesis that baseline scores on ASI subscales would predict posttreatment OCD symptom severity above and beyond baseline OCD and depressive symptom severity, we computed a hierarchical linear regression model. Results are shown in Table 3.

In Step 1, baseline DOCS (to control for pretreatment OCD severity) and BDI-II (to control for depressive symptom severity) scores jointly explained a significant amount of variance (28.62\%) in posttreatment DOCS scores, $F(2,106)=21.276, p<0.001$. Adding ASI subscale scores in Step 2 of this model account for statistically significant additional variance $\left(\mathrm{R}^{2}\right.$ change $\left.=0.05, p=0.049\right)$, such that higher pretreatment AS predicted poorer OCD treatment response even after controlling for pretreatment OCD and depression severity. However, after accounting for pretreatment DOCS and BDI-II scores, individual ASI subscales did not uniquely predict significant variance in posttreatment DOCS scores (i.e., ASI subscales only jointly explained significant outcome variance).

Based on our findings that depressive symptoms were only moderately correlated with baseline and posttreatment DOCS scores, we conducted an exploratory hierarchical regression excluding BDI-II scores, such that baseline DOCS were entered in Step 1, and the ASI subscales were entered in Step 2 of the model predicting posttreatment DOCS scores. The pattern of results was generally consistent with the primary analysis. Specifically, pretreatment OCD symptoms explained for a significant amount $(27.2 \%)$ of posttreatment OCD symptoms in Step $1, F(1,108)=41.63$, $p<0.001$, yet the addition of the ASI subscales in Step 2 accounted for an additional $6.0 \%$ of outcome variance, $F_{\Delta R}^{2}(3,105)=3.15$, $p=0.028$. In this simplified model, the ASI physical subscale accounted for a marginally significant amount of unique outcome variance, $\beta=0.198, t(104)=1.83, p=0.070, s p r^{2}=0.021$.

\section{Discussion}

It is important to understand why certain OCD patients do not adhere or respond to CBT, the gold-standard intervention for OCD. In this vein, trait levels of AS represent a relevant and promising predictor of treatment outcome. Accordingly, the current study examined the extent to which baseline AS predicted treatment outcome in a sample of individuals with a clinical diagnosis of OCD undergoing CBT. Consistent with our first hypothesis, AS was positively correlated with baseline OCD severity. This is in line with previous work (see Robinson \& Freeston, 2014) and indicates that increased fear of arousal-related body sensations is associated with increased OCD symptom severity. Our second hypothesis, that greater baseline levels of AS would prospectively predict higher posttreatment OCD symptom severity after controlling for pretreatment OCD and depression severity (i.e., factors consistently documented to influence $O C D$ treatment response), was

Table 3

Hierarchical linear regression predicting posttreatment DOCS total scores.

\begin{tabular}{llllllll}
\hline & $\Delta R^{2}$ & $B$ & $S E_{\mathrm{B}}$ & $\beta$ & $t$ & $p$ & $s r^{2}$ \\
\hline Step 1 & 0.29 & & & & & $<0.001$ & \\
\hline Baseline DOCS & & 0.41 & 0.07 & 0.50 & 5.63 & $<0.001$ & 0.213 \\
Baseline BDI-II & & 0.06 & 0.08 & 0.08 & 0.85 & 0.397 & 0.005 \\
\hline Step 2 & 0.05 & & & & & 0.049 & \\
\hline Baseline DOCS & & 0.35 & 0.08 & 0.43 & 4.63 & $<0.001$ & 0.138 \\
Baseline BDI-II & & -0.02 & 0.09 & -0.02 & -0.19 & 0.853 & $<0.001$ \\
ASI-Physical & & 0.42 & 0.23 & 0.20 & 1.79 & 0.077 & 0.021 \\
ASI-Mental & & 0.64 & 0.46 & 0.17 & 1.40 & 0.166 & 0.013 \\
ASI-Social & & -0.15 & 0.28 & -0.07 & -0.54 & 0.590 & 0.002 \\
\hline
\end{tabular}

DOCS $=$ Dimensional Obsessive-Compulsive Scale; BDI-II = Beck Depression Inventory-II; ASI = Anxiety Sensitivity Index; $s r^{2}=$ squared semipartial correlation. also supported. Even after controlling for pretreatment OCD and depression severity (which did not significantly predict CBT treatment outcome in our sample), the construct of AS accounted for significant, incremental variance in posttreatment OCD severity; although, no individual AS dimension emerged as a unique predictor. This suggests that the fear of anxious arousal in general predicts the outcome of CBT for OCD, and therefore represents a promising target for enhancing treatment response.

An important next step is to consider the mechanism through which AS hinders the effects of CBT for OCD. Although it was not measured directly in the present study, we hypothesize that elevated AS served to amplify exposure difficulty. Specifically, exposures that generated sensations of anxious arousal may have been experienced as more challenging and/or distressing not only because of the fear of the stimuli themselves (e.g., "Touching the toilet will make me sick"), but also because of the threat associated with physiological arousal (e.g., "If my heart races, I will have a heart attack"). In effect, OCD patients with high AS are simultaneously confronting two conditioned fear stimuli during exposures, which might understandably lead to nonadherence with prescribed exposure tasks. To test this hypothesis, future work examining AS as an outcome predictor might include measures of the quality of engagement in ERP as well as measures of physiological arousal during exposures (e.g., heart rate and skin conductance).

Regarding clinical implications of our findings, it may be beneficial to first conduct interoceptive exposure (IE; deliberately inducing feared-yet-safe body sensations without engaging in arousal-reduction strategies) with high AS patients prior to exposures to OCD-related stimuli with the aim of extinguishing the fear of arousal-related sensations. This might increase willingness to comply with ERP procedures for OCD. Consistent with inhibitory learning approaches to fear extinction, CBT might also involve the simultaneous use of IE and in vivo exposure to optimize consolidation of long-term extinction learning (e.g., Arch \& Abramowitz, 2015; Craske et al., 2008, 2014). For example, a patient who interprets her trembling hands as indicating that she is highly likely to act on her unwanted obsessional thoughts of stabbing a loved one might conduct tailored IE exercises (i.e., holding a pushup position to induce trembling) immediately prior to holding a knife while near a loved one (in vivo exposure). This procedure is consistent with the principle of deepened extinction as described by Rescorla (2006), in which multiple fear cues are combined during exposure.

Strengths of the present study included the large clinical sample (which maximizes power) as well as the variability of patient symptomatology (which offers greater external validity and sample generalizability than many RCTs). On the other hand, one limitation of this research was the use of the original ASI (Reiss et al., 1986), rather than the newer ASI-3 (Taylor et al., 2007). Second, assessments relied on self-report measures administered at pre- and posttreatment only; future research should administer assessments at additional time points, as well as include clinician-rated assessments of symptom severity. A third limitation regards the differences in treatment duration and multimodal intervention at Rogers Memorial Hospital, as well as the fact that some participants may have had medication adjustments during treatment (if deemed appropriate by the on-site psychiatrist). Along these lines, it is also possible that patients in our sample did conduct OCD exposures that in some way addressed their AS concerns. Finally, because our sample was receiving residential treatment, our findings may not apply to routine clinical settings. Thus, future research examining the role of particular dimensions of AS in various approaches of standardized CBT for OCD should be conducted. 


\section{Acknowledgements, declaration of interest, and role of funding organizations}

The authors have no conflicts of interest to disclose. The authors did not receive support from funding organizations to conduct this research. The authors assert that all procedures contributing to this work comply with the ethical standards of the relevant national and institutional committees on human experimentation and with the Helsinki Declaration of 1975, as revised in 2008.

\section{References}

Abramowitz, J. S., Deacon, B., Olatunji, B., Wheaton, M. G., Berman, N., Losardo, D. et al. (2010). Assessment of obsessive-compulsive symptom dimensions: Development and evaluation of the dimensional obsessive-compulsive scale. Psychological Assessment, 22, 180-198.

Abramowitz, J. S., \& Foa, E. B. (2000). Does comorbid major depressive disorder influence out-come of exposure and response prevention for OCD? Behavio Therapy, 31, 795-800.

Abramowitz, J. S., Franklin, M. E., Kozak, M. J., Street, G. P., \& Foa, E. B. (2000). The effects of pretreatment depression on cognitive-behavioral treatment outcome in OCD clinic patients. Behavior Therapy, 31, 517-528.

American Psychiatric Association (APA). (2000). Diagnostic and statistical manual of mental disorders (4th ed., text rev.). Washington, DC: Author.

American Psychiatric Association (APA). (2013). Diagnostic and statistical manual of mental disorders ( (5th ed.). Washington, DC: Author.

Anholt, G. E., Aderka, I. M., van Balkom, A. M., Smit, J. H., Hermesh, H., et al. (2011). The impact of depression on the treatment of obsessive-compulsive disorder: Results from a 5-year follow-up. Journal of Affective Disorders, 135, 201-207. http://dx.doi.org/10.1016/j.jad.2011.07.018.

Arch, J. J., \& Abramowitz, J. S. (2015). Exposure therapy for obsessive-compulsive disorder: An optimizing inhibitory learning approach. Journal of Obsessivecompulsive and Related Disorders, 6, 174-182.

Beck, A. T., Steer, R. A., \& Brown, G. K. (1996). Manual for the Beck depression inventory-II. San Antonio, TX: Psychological Corporation.

Calamari, J. E., Rector, N. A., Woodard, J. L., Cohen, R. J., \& Chik, H. M. (2008). Anxiety sensitivity and obsessive-compulsive disorder. Assessment, 15, 351-363.

Cox, B. J., Borger, S. C., \& Enns, M. W. (1999). Anxiety sensitivity and emotiona disorders: Psychometric studies and their theoretical implications. In S. Taylor (Ed.), Anxiety sensitivity: Theory, research, and treatment of the fear of anxiety (pp. 115-148). Mahwah, NJ, US: Lawrence Erlbaum Associates Publishers.

Craske, M. G., Kircanski, K., Zeilkowsky, M., Mystkowski, J., Chowdhury, N., \& Baker, A. (2008). Optimizing inhibitory learning during exposure therapy. Behaviour Research and Therapy, 46, 5-27.

Craske, M. G., Treanor, M., Conway, C. C., Zbozinek, T., \& Vervliet, B. (2014). Maximizing exposure therapy: An inhibitory learning approach. Behaviour Research and Therapy, 58, 10-23.

David, B., Olatunji, B. O., Armstrong, T., Ciesielski, B. G., Bondy, C. L., \& BromanFulks, J. (2009). Incremental specificity of disgust sensitivity in the prediction of obsessive-compulsive disorder symptoms: Cross-sectional and prospective approaches. Journal of Behavior Therapy and Experimental Psychiatry, 40, $533-543$.

Deacon, B. J. \& Abramowitz, J. S. (2006). Anxiety sensitivity and its dimensions across the anxiety disorders. Journal of Anxiety Disorders, 20, 837-857.

Farrell, N. R., Ouimet, A. J., Rowa, K., Soreni, N., Swinson, R. P., \& McCabe, R. E. (2016) Who gets better when? An investigation of change patterns in group cognitive behavioral therapy for obsessive-compulsive disorder. Journal of Obsessivecompulsive and Related Disorders, 10, 35-41.

Foa, E. B., Grayson, J. B., Steketee, G. S., Doppelt, H. G., Turner, R. M., \& Latimer, P. R (1983). Success and failure in the behavioral treatment of obsessive-compulsives. Journal of Consulting and Clinical Psychology, 51, 287-297.

Goodman, W. K., Price, L. H., Rasmussen, S. A., \& Mazure, C. (1989a). The yale-brown obsessive compulsive scale: I. Development, use, and reliability. Archives of General Psychiatry, 46, 1006-1011.

Goodman, W. K., Price, L. H., Rasmussen, S. A., \& Mazure, C. (1989b). The yale-brown obsessive compulsive scale: II. Validity. Archives of General Psychiatry, 46,
1012-1016.

Keough, M. E., Riccardi, C. J., Timpano, K. R., Mitchell, M. A., \& Schmidt, N. B. (2010) Anxiety symptomatology: The association with distress tolerance and anxiety sensitivity. Behavior Therapy, 41, 567-574.

Kessler, R. C., Berglund, P., Demler, O., Jin, R., Merikangas, K. R., \& Walters, E. E. (2005). Lifetime prevalence and age-of-onset distributions of DSM-IV disorders in the national comorbidity Survey replication. Archives of General Psychiatry, 62, 593-602.

Knopp, J. Knowles, S., Bee, P. Lovell, K., \& Bower, P. (2013). A systematic review of predictors and moderators of response to psychological therapies in OCD: Do we have enough empirical evidence to target treatment? Clinical Psychology Review, 33, 1067-1081.

Kozak, M. J., \& Foa, E. B. (1997). Mastery of obsessive-compulsive disorder: A cognitive-behavioral approach. San Antonio, TX: The Psychological Corporation.

Laposa, J. M., Collimore, K. C., Hawley, L. L., \& Rector, N. A. (2015). Distress tolerance in OCD and anxiety disorders, and its relationship with anxiety sensitivity and intolerance of uncertainty. Journal of Anxiety Disorders, 33, 8-14.

Norton, P. J., Sexton, K. A., Walker, J. R., \& Norton, G. R. (2005). Hierarchical model of vulnerabilities for anxiety: Replication and extension with a clinical sample. Cognitive Behavior Therapy, 34, 50-63.

Olatunji, B. O., Cisler, J. M., \& Deacon, B. J. (2010). Efficacy of cognitive behavioral therapy for anxiety disorders: A review of meta-analytic findings. Psychiatric Clinics of North America, 33, 557-577.

Peterson, R. A., \& Reiss, S. (1992). Anxiety sensitivity Index revised test manual (2nd ed.). Worthington, OH: Publishing Corporation.

Rachman, S. (1997). A cognitive theory of obsessions. Behaviour Research and Therapy, 35, 793-802.

Rachman, S. (1998). A cognitive theory of obsessions: Elaborations. Behaviour Research and Therapy, 36, 385-401.

Reiss, S., \& McNally, R. J. (1985). The expectancy model of fear. In S. Reiss, \& R. R. Bootzin (Eds.), Theoretical issues in behavior therapy (pp. 107-121). London, England: Academic Press.

Reiss, S., Peterson, R. A., Gursky, D. M., \& McNally, R. J. (1986). Anxiety sensitivity, anxiety frequency and the prediction of fearfulness. Behavior Research and Therapy, 24, $1-8$.

Rescorla, R. A. (2006). Deepened extinction from compound stimulus presentation. Journal of Experimental Psychology: Animal Behavior Processes, 32, 135-144.

Robinson, L. J., \& Freeston, M. H. (2014). Emotion and internal experience in Obsessive Compulsive Disorder: Reviewing the role of alexithymia, anxiety sensitivity and distress tolerance. Clinical Psychology Review, 34, 256-271.

Schmidt, N. B., Zvolensky, M. J., \& Maner, J. K. (2006). Anxiety sensitivity: Prospective prediction of panic attacks and Axis I pathology. Journal of Psychiatric Research, 40, 691-699.

Sexton, K. A., Norton, P. J., Walker, J. R., \& Norton, G. R. (2003). Hierarchical model of generalized and specific vulnerabilities in anxiety. Cognitive Behavior Therapy, $32,82-94$

Steketee, G., Chambless, D. L., \& Tran, G. Q. (2001). Effects of axis I and II comorbidity on behavior therapy outcome for obsessive-compulsive disorder and agoraphobia. Comprehensive Psychiatry, 42, 76-86.

Taylor, S. (1999). Anxiety sensitivity: Theory, research, and treatment of the fear of anxiety. Mahwah, NJ: Lawrence Erlbaum Associates.

Taylor, S., Koch, W. J., Woody, S., \& McLean, P. (1996). Anxiety sensitivity and depression: How are they related? Journal of Abnormal Psychology, 105, 474-479.

Taylor, S., Zvolensky, M. J., Cox, B. J., Deacon, B., Heimberg, R. G., Ledley, D. R., ... Coles, M. (2007). Robust dimensions of anxiety sensitivity: Development and initial validation of the anxiety sensitivity Index-3. Psychological Assessment, 19, 176.

Wheaton, M. G., Deacon, B. J., McGrath, P. B., Berman, N. C., \& Abramowitz, J. S. (2012). Dimensions of anxiety sensitivity in the anxiety disorders: Evaluation of the anxiety sensitivity index-third version. Journal of Anxiety Disorders, 26, 401-408.

Wheaton, M. G., Mahaffey, B., Timpano, K. R., Berman, N. C., \& Abramowitz, J. S. (2012). The relationship between anxiety sensitivity and obsessive-compulsive symptom dimensions. Journal of Behavior Therapy and Experimental Psychiatry, 43, 891-896.

Zinbarg, R. E., Barlow, D. H., \& Brown, T. A. (1997). Hierarchical structure and general factor saturation of the anxiety sensitivity index: Evidence and implications. Psychological Assessment, 9, 277-284. 